\title{
Special issue of Supercomputing Journal on secure, manageable and controllable grid services
}

\author{
Christophe Cérin · Jean-Luc Gaudiot • \\ Kuan-Ching Li
}

Published online: 12 June 2009

(C) Springer Science+Business Media, LLC 2009

\section{Introduction}

After a decade of visible success stories in building clusters, then cluster of clusters, what we call "Grid systems" are expected to connect large numbers of heterogeneous resources (PC, databases, HPC clusters, instruments, sensors, visualization tools, etc.), to be accessed by many users, to execute a large variety of applications (number crunching, data access, multimedia, etc.) and to deal with many scientific fields (health, economy, computing, etc.). Such context forces applications to work in a dynamically changing computing environment, which gives rise to approaches to master complexity that are radically different from those found in traditional computing environments.

An architecture (in the sense of 'hardware') alone is not sufficient to ensure an efficient use of resources and as with other fields in computing research, we need also to focus on middleware, languages, to cite but a few of the challenges. We considered first that large-scale systems such as Grids are devoted to exploit parallelism in applications, may entail the coordination of large numbers of processing elements, the synchronization and communication in the larger scale, the programming models and

C. Cérin $(\bowtie)$

University of Paris 13, Villetaneuse, France

e-mail: Christophe.Cerin@lipn.univ-paris13.fr

J.-L. Gaudiot

University of California, Irvine, CA, USA

e-mail: gaudiot@uci.edu

K.-C. $\mathrm{Li}$

Providence University, Taichung, Taiwan, ROC

e-mail: kuancli@pu.edu.tw 
productivity. After some refinement steps, we decided that this special issue would focus on issues of security and manageable grid services, meaning that we are interested in middleware and distributed contexts that offer some guarantees on security (in a broad sense) and are manageable because we need to deploy them on a large collection of resources. We still consider that such important topics are not very well covered in the literature.

Grids are distributed systems and like for them, the notion of security, the way we manage such large systems and the way we control the Grid system are of particular interest. For instance, 'controllable' means: how we measure the activity of the Grid and how we report it. In this context, 'manageable' means: how we deploy the Grid architecture, the Grid software, and how we start jobs (under controllable events such as the availability of resources).

The 'security' term refers to the traditional fields of authentication and fault tolerance, but means safe execution (how to certify results, how to adapt computation according to some metric). Moreover, it seems natural that all of these services should collaborate making the building of middleware a challenging problem.

The idea of this special issue, based on the ideas explained in the previous paragraph, was announced during GPC2007 in Paris and we started to refine the topics during the event. Please refer to http://www-lipn.univ-paris13.fr/GPC2007/ for information about GPC2007 (the proceedings were published with Springer here: http://www.springer.com/computer/foundations/book/978-3-540-72359-2). This special issue was dedicated at gathering papers in the fields of Grid / Distributed systems. Contributions in such connected fields as P2P systems, Sensors networks, Networking, Large-scale heterogeneous distributed databases, were also sought.

Topics

We invited authors to submit original and unpublished work. We also required that manuscript be not concurrently submitted to another journal or conference. We decided to accept 'extended work' but only if it included some original/new insights. Papers were reviewed by three experts. Finally, we informed authors that papers without performance evaluation (either theoretical or experimental) would not be considered. In other words, papers should be more than 'position papers' or a small increment from a previous publication.

Hence, we solicited novel papers on a broad range of topics that included, but were not limited to, the following keywords:

- Grid monitoring and controlling (forecasting ...) systems

- Grid management systems (deployment of infrastructures and applications)

- Grid security (authentication, adaptation, results' certification)

- Aggregation of secure/manageable components into Grid middleware

- Algorithms related to resource brokers, load balancing and heterogeneity

- Applications and experiences with secure/manageable/controllable Grid infrastructures.

\section{Selected papers}

We received 37 submissions and we selected the following 7 outstanding papers based on the reviews of at least 3 reviews per paper: 
"Ant Colony Optimization Inspired Resource Discovery in P2P Grid Systems" by Yuhui Deng et al. The paper presents a resource-discovering algorithm for Grid system based using ant colony optimization. After presenting the proposed algorithm, the authors present experimental results based on simulation to show the performance results. The experimental results are based on simulation and show a better hit ratio compared to the hash algorithm, at the cost of longer average hops.

Rodrigo Mello et al. The authors introduce an interesting idea of tackling the dynamic process scheduling problem by application of prediction techniques based on chaos and fractal theory and nonlinear techniques on time series. The results are encouraging and demonstrate the viability of the approach.

"QoS-Aware, Access-Efficient and Storage-Efficient Replica Placement in Grid Environments" by Jan-Jan Wu et al. The paper addresses the QoS-aware replica placement problem in the Grid. The contribution of the paper is that it improves previous approaches by taking into account additional aspects, such as the storage cost, update cost, access cost and the capacity of each replica server. The paper presents two algorithms: 'greedy remove' and 'greedy add' and compares them, between them, and against a random solution.

"Advanced Service Trading for Scientific Computing over the Grid" by Aurelie Herault et al. This paper presents an alternative to the use of ontologies for trading in SOA (Service Oriented Architecture). The authors use algebraic semantic which is a well-known tool in parallel computing models. The main advantage against ontologies is that it is easier for specialists to write an algebraic semantics and that in some cases trading can be proven decidable.

"Building Effective Mutual Exclusion Services for Grids" by Julien Sopena et al. The authors propose algorithms for mutual exclusion service in Grid environments, where application processes are over several clusters interconnected by long distance links. The algorithms are executed on Grid'5000, which is a large-scale grid experimental testbed. The results are thoroughly investigated.

"Efficient Integration of Fine-Grained Access Control and Resource Brokering in Grid" by B. Crispo et al. In this paper, the authors present a resource brokering service for Grid systems, which considers authorization policies of the Grid nodes in the process of selecting the resources to be assigned to a request. It is based on the concept of fine-grained access control (FGAC) which enables participating Grid nodes to specify fine-grained policies concerning the conditions under which Grid clients can access their resources.

The authors present Group-By Rule, a novel method for policy organization and compare its performance with other strategies.

"Liana: A Decentralized Load-Dependent Scheduler for Performance-Cost Optimization of Grid Service" by Dan Liu et al. In this paper the authors propose a scalable decentralized scheduler called Liana.

The scheduler operates on a service-oriented overlay network and its algorithm is based on a degree-driven protocol. The goal of this tool achieves a close-to-optimal service utilization by minimizing the average cost that depends on the Grid system load. So, the paper is related to scheduling based on heuristics and a smart queuing network. 